\title{
Gender-specific distribution of mefloquine in the blood following the administration of therapeutic doses
}

\author{
Walther H Wernsdorfer ${ }^{1}$, Harald Noedl ${ }^{1}$, Pamela Rendi-Wagner ${ }^{1}$, Herwig Kollaritsch', Gerhard Wiedermann ${ }^{1}$, \\ Andrea Mikolasek', Juntra Karbwang ${ }^{2}$ and Kesara Na-Bangchang ${ }^{3 *}$
}

\begin{abstract}
Background: The objectives of the study were to elucidate the gender-specific distribution of mefloquine in cellular and fluid blood compartments when given at therapeutic dosage, to assess its correlation with the occurrence of treatment-related adverse events, and to explore the necessity of adjusting treatment guidelines for females.

Methods: The distribution of mefloquine following the administration of standard therapeutic doses $(1,250 \mathrm{mg}$ mefloquine in split dose) to 22 healthy Caucasian volunteers was assessed in whole blood, serum, plasma, red blood cells (RBCs), white blood cells, and platelets using high performance liquid chromatography.

Results: Plasma mefloquine concentrations after 14 hours were considerably higher in female subjects than in males (2,778 vs $1,017 \mathrm{ng} / \mathrm{ml}$ at H14), concordant with a significantly higher frequency, duration, and severity of adverse reactions. However, mean drug concentrations of RBC appeared slightly higher in male volunteers (857 vs $719 \mathrm{ng} / \mathrm{ml}$ ). At H48, a similar situation prevailed, and at H168 the mefloquine concentrations in plasma continued to be higher in females compared to males $(1,353$ vs $666 \mathrm{ng} / \mathrm{ml})$, while the concentrations of RBC were similar in females (389 vs $375 \mathrm{ng} / \mathrm{ml}$ ). Since the observations relate to healthy individuals, they do not take into account selective uptake of mefloquine by Plasmodium-infected erythrocytes as in the case of therapeutic drug use.

Conclusion: Although plasma mefloquine concentrations in female healthy volunteers are considerably higher and the concentrations of the RBCs are initially lower compared to males, they do not seem to justify an adjustment of treatment guidelines for mefloquine in female Caucasian individuals.
\end{abstract}

Keywords: Mefloquine, Pharmacokinetics, Gender-specific distribution, Plasma, Red blood cell

\section{Background}

With the emergence and spread of multi-drug resistance Plasmodium falciparum, mefloquine is currently being used as a combination partner with artesunate in the artemisinin-based combination therapy (ACT). Mefloquine resistance and the resultant drop in malaria cure rates in Southeast Asia has necessitated increases in the administered mefloquine from $15 \mathrm{mg} / \mathrm{kg}$ as a single dose to $25 \mathrm{mg} / \mathrm{kg}$ in split dose, and also in combination with artesunate treatment [1]. The apparent incidence of adverse events (AEs) following the oral administration of

\footnotetext{
* Correspondence: kesaratmu@yahoo.com

${ }^{3}$ Chulabhorn International College of Medicine, Thammasat University, Rangsit Campus, Pathumthani, Thailand

Full list of author information is available at the end of the article
}

mefloquine is high, with reports of 47 to $90 \%$ of adults experiencing some type of AEs [2-4]. The use of high doses of mefloquine is also associated with higher frequencies of AEs, particularly in female patients $[1,5,6]$.

Mefloquine [2,8-bis(trifluoromethyl)quinolin-4-yl]-(2piperidyl)methanol) was discovered by the Walter Reed Army Institute of Research in the 1970s. Mefloquine is a synthetic quinine analogue. The anti-malarial effects of mefloquine are believed to be due to its accumulation within the parasite's food vacuole where it interacts with haem. In this way, the formation of the haemozoin polymer is prevented and the subsequent drug-haem complex confers toxicity towards malaria parasites [2]. Mefloquine also inhibits acetylcholinesterase and butyrylcholinesterase, the likely cause for the frequent 
gastrointestinal and central nervous systems-related AEs which occur at high dosages of the drug [7]. As mefloquine hydrochloride is rapidly absorbed by the gastrointestinal (GI) tract, leading to a sudden onset of side effects, it is manufactured as a number of different formulations geared towards slower absorption in the GI tract. Considerable differences in mefloquine bioavailability have been reported for the various formulations $[8,9]$. Following oral administration of Lariam ${ }^{\odot}$ tablets, approximately $75-80 \%$ of the drug is absorbed, and time-tomaximum concentration $\left(t_{\max }\right)$ values were found to be 2 to 12 hours. There is little pre-systemic metabolism of the compound, which has a plasma half-life of 15 to 33 days, with a mean of 21.4 days [10]. Mefloquine is quickly distributed throughout the body, and has a high affinity for lipids. In blood plasma, mefloquine is substantially protein-bound. The majority of mefloquine is metabolized by the liver to produce carboxy-mefloquine, which has no anti-malarial activity and a toxicity and half-life similar to the parent molecule [11]. Despite being highly soluble, well distributed, and extensively tissue binding, reports of substantial binding of mefloquine to plasma proteins in volunteers and patients has also been documented [12].

The gender-specific differences in the frequency and severity of AEs experienced following the administration of mefloquine in prophylactic as well as therapeutic dosages may at least in part be attributable to different distribution patterns in liquid and cellular blood compartments. Therefore, the aim of the present study was to elucidate the gender-specific distribution of mefloquine in these compartments at therapeutic dosages of the drug in order to assess any relationship with the occurrence of treatment-related AEs. Furthermore, the eventual necessity of adjusting treatment guidelines in female patients was explored.

\section{Methods \\ Subjects}

This study was conducted with 22 healthy Caucasian volunteers (10 males, 12 females) aged 20 to 45 years (median age of 26) at the Institute of Specific Prophylaxis and Tropical Medicine, Medical University of Vienna. Written informed consent was obtained from all study participants and the study protocol was approved by the ethical review board at the University of Vienna.

\section{Clinical and laboratory investigations}

Physical examinations, blood chemistry (including complete blood counts (CBCs)), urinalysis and pregnancy tests were performed upon enrolment. Those reporting chronic medical (including neuropsychiatric) disorders or mefloquine intolerance were excluded from the study, in addition to those who were heavy smokers or pregnant.
CBCs were conducted at 14, 48, and 168 hours after the administration of the first mefloquine dose. Urinalysis, biochemistry analysis and complete physical examinations were repeated 168 hours after drug administration. All volunteers were monitored for AEs during the 21 days after the first dose had been administered. During the entire period the volunteers followed their normal daily activities.

\section{Drug administration}

All subjects received 1,250 mg Lariam ${ }^{\bullet}$ each (five tablets, $250 \mathrm{mg}$ mefloquine hydrochloride per tablet; Hoffmann-la Roche Pharmaceuticals, Basel, Switzerland) as split dosages of $750 \mathrm{mg}$ (three tablets) followed by another $500 \mathrm{mg}$ (two tablets) six hours later.

\section{Blood collection}

Venous blood samples were collected in sterile glass containers at 0 hours (immediately prior to drug administration), 14 hours (the estimated time to reach peak plasma concentrations, eight hours after the administration of the second mefloquine dose), 48 hours (estimated to be the beginning of the log-linear elimination phase), and 168 hours (the minimum time required for therapeutic drug levels to eliminate malaria parasites).

Whole blood samples were processed immediately by separating into six fractions (whole blood, plasma, serum, red blood cells (RBC), white blood cells (WBC), and platelets). This was done using both standard centrifugation methods as well as the use of Percoll $^{\circledR}$ (Amersham Biosciences, Buckinghamshire, UK) gradient centrifugation which utilizes colloidal silica coated with polyvinylpyrrolidone (at gradients 1.059 for platelets and 1.089 for leukocytes). To prevent mefloquine adhesion to plastic surfaces, all samples were stored in glass containers at $\leq-30^{\circ} \mathrm{C}$ until further analysis.

\section{Drug analysis}

Mefloquine concentrations were measured at the Pharmacology and Toxicology Unit, Chulaborn International College of Medicine, Thammasat University, Thailand, using high performance liquid chromatography (HPLC) with a Microbondapak C18 $(4.6 \times 250 \mathrm{~mm}$, particle size $5 \mu \mathrm{m})$, reverse-phase column and UV-detection at $222 \mathrm{~nm}$ [13]. The limit of quantification (LOQ) for the mefloquine assay is $2 \mathrm{ng} / \mathrm{ml}$. Drug concentrations were determined for whole blood, serum, plasma, RBCs, WBCs, and platelets.

\section{Statistical analysis}

The student t-test and the one way analysis of variance (ANOVA) were used to ascertain significant differences between group means. Correlations between two quantitative variables were investigated by means of Spearman 
correlation analysis. Non-parametric procedures were used for data not conforming to normal distribution. The level of statistical significance was set at $\alpha=0.05$ for all tests.

\section{Results}

The groups of male and female study participants were comparable in age (mean age 27.6 vs 26.6 years) with similar social backgrounds and prior exposure to anti-malarials. The mean total doses per $\mathrm{kg}$ of mefloquine received by the participants were 17.7 (range 13.89-20.83) and 20.7 (16.89-22.73) $\mathrm{mg} / \mathrm{kg}$ for healthy male (mean weight $71.4 \mathrm{~kg})$ and female $(61.2 \mathrm{~kg})$ volunteers, respectively.

Table 1 shows the mefloquine concentrations in whole blood, serum, plasma, RBCs, WBCs, and platelets from male and female subjects. Following the first dose, drug concentrations in whole blood in females reached $1,360 \mathrm{ng} / \mathrm{ml}$ at 14 hours to rise to $1,437 \mathrm{ng} / \mathrm{ml}$ at 48 hours, whereas male levels decreased moderately from 1,648 to $1,272 \mathrm{ng} / \mathrm{ml}$. At 168 hours, the drug in both males and females had decreased to approximately the same concentration in whole blood $(896 \mathrm{ng} / \mathrm{ml}$ in females vs $885 \mathrm{ng} / \mathrm{ml}$ in males). A significant difference $(p<0.001)$ between plasma concentrations in male $(1,017 \mathrm{ng} / \mathrm{ml})$ and female subjects $(2,778 \mathrm{ng} / \mathrm{ml})$ was found at 14 hours, whereas the concentration in whole blood was similar for both genders. While RBC drug concentrations were initially higher in male subjects, WBC and platelets levels were very similar. However, the gender differences in mefloquine concentrations in these cellular compartments were not found to be significant. RBC mefloquine levels were considerably lower than in whole blood or serum $(p<0.001)$, whereas platelet and WBC mefloquine levels were approximately six times higher and 20 times higher than in whole blood, respectively (both $p<0.001$ ). Mean levels in serum of females were lower than in plasma, whereas they were higher in males at H14 and H168.. However, there were no significant gender differences $(p>0.05)$. No correlation $(p>0.05)$ was seen between plasma drug

Table 1 Mean mefloquine concentrations, in $\mathrm{ng} / \mathrm{ml}$, in male $(n=10)$ and female $(n=12)$ healthy volunteers at 14 hours (H14), 48 hours (H48) and 168 hours (H168) after administration of the first drug dose

\begin{tabular}{|c|c|c|c|c|c|c|}
\hline \multirow[t]{2}{*}{ Blood } & \multicolumn{2}{|c|}{ H 14} & \multicolumn{2}{|c|}{ H 48} & \multicolumn{2}{|c|}{ H 168} \\
\hline & Female & Male & Female & Male & Female & Male \\
\hline Whole blood & 1,360 & 1,648 & 1,437 & 1,272 & 896 & 885 \\
\hline Serum & 1,570 & 1,623 & 1,244 & 847 & 811 & 1,304 \\
\hline Plasma & 2,778 & 1,017 & 2,106 & 1,214 & 1,353 & 666 \\
\hline $\mathrm{RBC}$ & 719 & 857 & 633 & 827 & 389 & 375 \\
\hline WBC & 35,641 & 33,885 & 32,414 & 29,447 & 17,584 & 16,214 \\
\hline Platelets & 9,212 & 9,002 & 9,710 & 7,825 & 5,538 & 5,808 \\
\hline
\end{tabular}

concentrations and RBC levels, suggesting that plasma levels are a poor predictor of RBC drug levels.

$\mathrm{RBC}$ mefloquine concentrations in both males and females and plasma mefloquine concentrations in males showed only slight changes between 14 and 48 hours. Plasma mefloquine concentrations in females, however, contrasted markedly with the other samples (Table 2). Concentrations of the drug in the plasma samples from females were much higher at 14 hours, and although a decrease occurred during the next 32 hours, concentrations of the drug were considerably higher than those of the other samples at each of the remaining time points.

All 22 subjects reported drug-related AEs. The most commonly reported AEs were vertigo (96\%), followed by nausea (82\%), headache (73\%), sleeping disturbances (59\%), and diarrhoea (41\%). The overall symptom scores (OSS) reflecting the frequency, duration and severity of drug-related AEs were significantly higher in female subjects (20.8 in males vs 43.3 in females; $p=0.003$ ). Frequency, duration and severity of AEs were directly correlated $(r=0.519 ; p=0.016)$ with plasma drug concentrations (Figure 1). No such correlation was found for drug concentrations in RBCs, WBCs and platelets $(p>0.05)$.

\section{Discussion}

Previous studies with mefloquine have shown that a significantly higher frequency and severity of treatmentand prophylaxis-related AEs occur in female patients [14-16]. Schwartz et al. [14] hypothesized that if AEs of mefloquine were related to blood concentrations, drug monitoring could minimize any untoward effects of the drug. However, these researchers were unable to find any gender-related differences in serum mefloquine concentrations. Kollaritsch et al. [6] reported a significantly higher $\mathrm{C}_{\max }$ with female plasma concentrations and higher incidences of AEs, subsequently recommending an adjustment of dose regimens in female mefloquine recipients.

The present study shows a distinct correlation between mefloquine plasma concentrations and the severity, duration and frequency of AEs. Higher mefloquine dosages, leading to elevated plasma concentrations, are generally considered to be the most influential parameter in the pharmacodynamic properties of a drug. AEs also generally coincide with high blood compartment drug concentrations $[1,14]$. However, the most important pharmacological parameter in terms of drug efficacy is the intra-erythrocytic drug concentration, since the erythrocyte cytoplasm is the target for malaria parasites. In pharmacokinetic studies, plasma or serum levels are typically the only parameters that are measured. Previous data suggest that although plasma concentrations are significantly higher in females, their RBC drug concentrations at $\mathrm{H} 14$ and $\mathrm{H} 48$ are even 
Table 2 Gender-specific statistical comparison of mean mefloquine concentrations in plasma and red blood cells $(\mathrm{ng} / \mathrm{ml})$, and of mean plasma/red blood cell ratios

\begin{tabular}{|c|c|c|c|c|c|c|c|c|}
\hline \multirow[t]{2}{*}{ Time } & \multicolumn{3}{|c|}{ Plasma } & \multicolumn{3}{|c|}{ RBC } & \multicolumn{2}{|c|}{ Mean Plasma/RBC } \\
\hline & Female & Male & $p^{*}$ & Female & Male & $p^{*}$ & Female/Male & $p^{*}$ \\
\hline H 14 & 2,778 & 1,017 & $<0.001$ & 719 & 857 & $>0.05$ & 3.51 & $<0.01$ \\
\hline H 48 & 2,106 & 1,214 & $<0.05$ & 633 & 827 & $>0.05$ & 2.84 & $<0.05$ \\
\hline H 168 & 1,353 & 666 & $<0.05$ & 389 & 375 & $>0.05$ & 2.75 & $>0.05$ \\
\hline
\end{tabular}

*Student $t$-test.

slightly lower than in males. Since the erythrocytes are the site of malarial infection, this may be interpreted as a potential shortfall of therapeutic activity [17]. However, previous investigationshave shown that erythrocytes infected with $P$. falciparum contain over four times as much mefloquine as compared to non-infected RBC, a phenomenon similar to the selective uptake of chloroquine by parasitized erythrocytes $[18,19]$. These observations would also explain the equivalence of the therapeutic efficacy of mefloquine in uncomplicated infections with mefloquine-sensitive $P$. falciparum in both genders.

The lack of any significant correlation between plasma and erythrocytic drug concentrations suggests that mefloquine plasma levels may not truly represent the amount of drug reaching uninfected or parasitized RBCs. However, this would also mean that low plasma concentrations, as observed in predominantly male populations, do not automatically indicate sub-therapeutic erythrocytic drug concentrations [20].

The yardstick used in assessing therapeutic drug regimens of mefloquine in the ACT is the threshold of therapeutic efficacy. This is obviously not a static parameter since it is likely to vary with the sensitivity of the malaria parasite. The plasma concentrations that have generally been considered as prophylactic or therapeutic in the past seem now to require reassessment. The lack of a correlation between plasma and erythrocytic drug concentrations points in the same direction. However, in view of the selective uptake of mefloquine by infected erythrocytes, the significance of mefloquine bound to uninfected RBC becomes questionable. Thus, the reported prophylactic threshold of $400 \mathrm{ng}$ mefloquine per $\mathrm{ml}[21]$ or $567 \mathrm{ng} / \mathrm{ml}(1.5 \mu \mathrm{M} / \mathrm{l})$ [22] in plasma seems to apply to situations where $P$. falciparum is not resistant to the drug, indicating that enough mefloquine reaches the parasites to kill them by this form of suppressive prophylaxis. It is noted that mefloquine is curently being used as a combination partner with artesunate in the artemisinin-based combination therapy $(\mathrm{ACT})$. This is in contrast to its use as a prophylactic where adverse effects are rather more relevant as the drug is being given to a health individual.

The most commonly reported AEs were vertigo, nausea, headache, sleep disturbances, and diarrhoea, with a

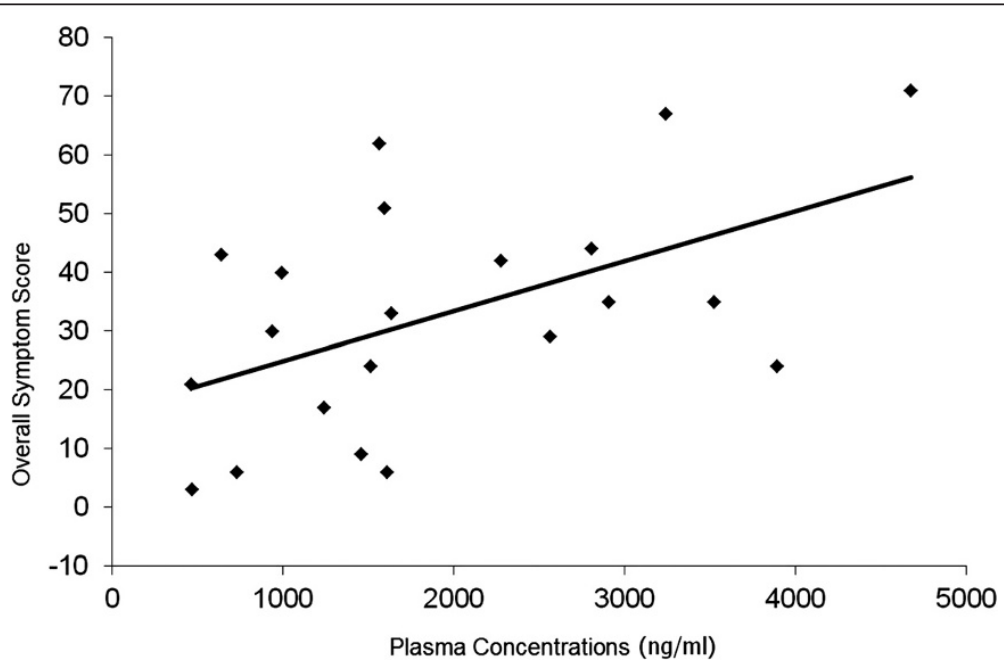

Figure 1 Scatter plot and regression line for mefloquine plasma concentrations $(\mathbf{n g} / \mathrm{ml})$ and overall symptom score. The frequency, duration and severity of adverse events (represented by OSS) exhibited significant correlation with mefloquine plasma levels $(y=0.0085 x+16.465$, $r=0.519 ; p=0.016)$. 
significantly higher frequency, duration and severity of drug-related AEs in women. These findings confirm previous observations in healthy adults [23]. Far fewer AEs are generally reported from clinical studies in falciparum malaria patients. The similarity between symptoms of malaria and some common drug-induced AEs may be the cause of this phenomenon [2]. In addition, the most frequent central nervous system-related $\mathrm{AE}$, vertigo, may be less frequently noticed by patients confined to bed-rest.

Mefloquine concentrations were much higher in WBCs and platelets, suggesting an active uptake of the drug into these cells. However, these levels appear to have little relevance for the treatment of malaria. The considerable differences between concentrations in plasma and serum levels may indicate the involvement of differential binding to specific proteins in the distribution of mefloquine, eg, fibrinogen [12]. In a previous study in healthy subjects however, mefloquine concentration was found to be higher in serum compared to whole blood [24].

\section{Conclusions}

The higher $\mathrm{AE}$ frequencies and severities caused by higher plasma concentrations in females in combination with lower $\mathrm{RBC}$ drug concentrations create speculation about the risks and benefits of mefloquine treatment for female patients. In spite of the considerably higher number of AEs in females, a down-adjustment of mefloquine treatment guidelines for these individuals is not recommended. The observations should stimulate investigations of the mefloquine dynamics within the first compartment, especially movement between plasma and erythrocytes in normal blood and blood infected with $P$. falciparum. In this context it would also be important to determine the location of mefloquine on the surface and within uninfected and infected erythrocytes, complemented by a study of the relevant transport mechanisms.

\section{Competing interests}

The authors have declared that they have no competing interests.

\section{Authors' contributions}

WHW, JK and KN conceived and designed the experiments. HN, PR-W, HK, GW and AM performed the experiments. HN and HK analysed the data. WHW and KN-B wrote the paper. All authors read and approved the final manuscript.

\section{Acknowledgements}

The study was supported by University of Vienna, Austria, and Commission on Higher Education, Ministry of Education of Thailand.

\section{Author details}

'Institute of Specific Prophylaxis and Tropical Medicine, Centre for Physiology and Pathophysiology, Medical University of Vienna, Vienna, Austria. ${ }^{2}$ Department of Clinical Product Development, Nagasaki Institute of Tropical Medicine, Nagasaki, Japan. ${ }^{3}$ Chulabhorn International College of Medicine, Thammasat University, Rangsit Campus, Pathumthani, Thailand.

Received: 29 September 2013 Accepted: 5 December 2013 Published: 9 December 2013

\section{References}

1. Na-Bangchang K, Karbwang J: Current status of malaria chemotherapy and the role of pharmacology in antimalarial drug research and development. Fundam Clin Pharmacol 2009, 23:387-409.

2. Palmer KJ, Holliday SM, Brogden RN: Mefloquine. A review of its antimalarial activity, pharmacokinetic properties and therapeutic efficacy. Drugs 1993, 45:430-475.

3. Barrett PJ, Emmins PD, Clarke PD, Bradley DJ: Comparison of adverse events associated with use of mefloquine and combination of chloroquine and proguanil as antimalarial prophylaxis: postal and telephone survey of travellers. BMJ 1996, 313:525-528.

4. Croft AMJ, Garner P: Mefloquine to prevent malaria: a systemic review of clinical trials. BMJ 1997, 315:1412-1416.

5. Rendi-Wagner $P$, Noedl $H$, Wernsdorfer WH, Wiedermann $G$, Mikolasek $A$, Kollaritsch $\mathrm{H}$ : Unexpected frequency, duration and spectrum of adverse events after therapeutic dose of mefloquine in healthy adults. Acta Trop 2002, 81:167-173.

6. Kollaritsch H, Karbwang J, Wiedermann G, Mikolasek A, Na-Bangchang K, Wernsdorfer WH: Mefloquine concentration profiles during prophylactic dose regimens. Wien Klin Wochenschr 2000, 112:441-447.

7. Lim LY, Go ML: The anticholinesterase activity of mefloquine. Clin Exp Pharmacol Physiol 1985, 12:527-531.

8. Na-Bangchang K, Karbwang J, Palacios PA, Ubalee R, Saengtertsilapachai S, Wernsdorfer WH: Pharmacokinetics and bioequivalence evaluation of three commercial tablet formulations of mefloquine when given in combination with dihydroartemisinin in patients with acute uncomplicated falciparum malaria. Eur $J$ Clin Pharmacol 2000, 55:743-748.

9. Weidekamm E, Rusing G, Caplain H, Sorgel F, Crevoisier C: Lack of bioequivalence of a generic mefloquine tablet with the standard product. Eur J Clin Pharmacol 1998, 54:615-619.

10. Karbwang J, White NJ: Clinical pharmacokinetics of mefloquine. Clin Pharmacokinet 1990, 19:264-279.

11. Wernsdorfer WH: Antimalarial drugs. Health Co-operation Papers 1997, 15:151-198.

12. Looareesuwan $S$, White NJ, Warrell DA, Forgo I, Dubach UG, Ranalder UB, Schwartz DE: Studies of mefloquine bioavailability and kinetics using a stable isotope technique: a comparison of Thai patients with falciparum malaria and healthy Caucasian volunteers. Br J Clin Pharmacol 1987, 24:37-42.

13. Karbwang J, Molunto P, Na-Bangchang K, Bunnag D: Determination of mefloquine in biological fluids using high performance liquid chromatography. Southeast Asian J Trop Med Public Health 1989, 20:55-60.

14. Schwartz E, Potasman I, Rotenberg M, Almog S, Sadetzki S: Serious adverse events of mefloquine in relation to blood level and gender. Am J Trop Med Hyg 2001, 65:189-192.

15. Schlagenhauf $P$, Tschopp A, Johnson R, Nothdurft HD, Beck B, Schwartz E, Herold M, Krebs B, Veit O, Allwinn R, Steffen R: Tolerability of malaria chemoprophylaxis in non-immune travellers to sub-Saharan Africa: multicentre, randomised, double blind, four arm study. BMJ 2003, 327:1078.

16. Schlagenhauf $P$, Steffen R, Lobel H, Johnson R, Letz R, Tschopp A, Vranjes N, Bergqvist Y, Ericsson O, Hellgren U, Rombo L, Mannino S, Handschin J, Sturchler D: Mefloquine tolerability during chemoprophylaxis: focus on adverse event assessments, stereochemistry and compliance. Trop Med Int Health 1996, 1:485-494.

17. Seethorn $\mathrm{N}$, Wernsdorfer WH, Noedl H, Karbwang J, Na-Bangchang K: Investigation of the in vitro gender-specific partitioning of mefloquine in malarial infected red blood cells and plasma. Am J Trop Med Hyg 2013. Sep 3. [Epub ahead of print].

18. Diribe $C O$, Warhurst DC: A study of the uptake of chloroquine in malaria-infected erythrocytes. High and low affinity uptake and the influence of glucose and its analogues. Biochem Pharmacol 1985, 34:3019-3027.

19. Ginsburg H, Stein WD: Kinetic modelling of chloroquine uptake by malaria-infected erythrocytes. Assessment of the factors that may determine drug resistance. Biochem Pharmacol 1991, 41:1463-1470.

20. Schwartz E, Paul F, Pener H, Almog S, Rotenberg M, Golenser J: Malaria antibodies and mefloquine levels among United Nations troops in Angola. J Travel Med 2001, 8:113-116.

21. Lobel HO, Miani M, Eng T, Bernard KW, Hightower AW, Campbell CC: Long-term malaria prophylaxis with mefloquine. Lancet 1993, 341:848-851. 
22. World Health Organization: Advances in malaria chemotherapy. Report of a WHO Scientific Group. WHO Tech Rep Ser no. 711. WHO: Geneva; 1984.

23. Phillips MA, Kass RB: User acceptability patterns for mefloquine and doxycycline malaria chemoprophylaxis. J Travel Med 1996, 3:40-45.24.

24. Todd GD, Hopperus Buma AP, Green MD, Jaspers CA, Lobel HO: Comparison of whole blood and serum levels of mefloquine and its carboxylic acid metabolite. Am J Trop Med Hyg 1997, 57:399-402.

doi:10.1186/1475-2875-12-443

Cite this article as: Wernsdorfer et al:: Gender-specific distribution of mefloquine in the blood following the administration of therapeutic doses. Malaria Journal 2013 12:443.

\section{Submit your next manuscript to BioMed Central and take full advantage of:}

- Convenient online submission

- Thorough peer review

- No space constraints or color figure charges

- Immediate publication on acceptance

- Inclusion in PubMed, CAS, Scopus and Google Scholar

- Research which is freely available for redistribution 